\title{
Oral Submucous Fibrosis and Its Relation with Stromal Vascularity: A Systematic Review
}

\author{
Sayed Nishat Abdul
}

\begin{abstract}
Oral submucous fibrosis (OSMF) is a chronic progressive, scarring disease affecting oral, oropharyngeal, and sometimes the esophageal mucosa. It is characterized by the progressive fibrosis of the submucosal tissue. The pathogenesis of OSMF has been directly related to the habit of chewing areca nut and its commercial preparation, which is widespread in Indian subcontinent and Southeast Asia. Malignant transformation has been reported in $7 \%-12 \%$ cases of OSMF. Histopathological spectrum of OSMF includes the apparent alterations observed in the epithelium and connective tissue. Vascularity in OSMF has always been a matter of debate. The prevailing concept is that epithelial atrophy occurs due to lack of perfusion. The present systemic review was done to evaluate oral submucous fibrosis and its relation with stromal vascularity.
\end{abstract}

Index Terms-Oral Submucous Fibrosis, Vascularity, Epithelial Atrophy.

\section{INTRODUCTION}

OSMF is a premalignant condition of oral mucosa seen in individuals with habit of areca nut chewing. It is an insidious, chronic, irreversible progressive disease in which the symptoms vary from burning of oral mucosa to inability in opening mouth, difficulty in swallowing and speech. It is more prevalent in South East Asia and people of South Asian origin [1]. The majority of patients present with an intolerance to spicy food and rigidity of lips, buccal mucosa, tongue and palate leading to varying degrees of limitation of opening of mouth and tongue movements. It is characterized by mucosal rigidity of varying intensity with fibroelastic changes of juxtaepithelial layer that affects most parts of the oral cavity, pharynx and the upper third of the esophagus [2]. Pathogenesis is believed to involve juxta-epithelial inflammatory reaction and fibrosis in the oral mucosa, probably due to an increased cross-linking of collagen through up-regulation of lysyl oxidase activity. Fibrosis, or the building up of collagen, results from the effects of areca nut, which increases collagen production (e.g., stimulated by arecoline, an alkaloid) and decreases collagen degradation [3],[4]. Along with repair process, inflammation induced vascular response is noted in OSMF. Same sections of OSMF show varied vasculature scenario ranging from normal, dilated to constricted blood vessels and a combination of both is also seen. The narrowing of the vessels is seen initially in superficial mucosa and in later stages; it spreads to deeper connective tissue. Presence of dilated vessels in advanced stages of OSMF is reported; altogether making the vascularity in OSMF a matter of conjuncture [5].

Published on March 14, 2020.

\section{ORAL Submucous Fibrosis AND ITS RELATION WITH STROMAL VASCULARITY}

Various researchers have studied the mechanism of malignant transformation of OSMF. It is hypothesized that the dense fibrosis, epithelial atrophy and less vascularity, in the presence of an altered cytokine activity creates a unique environment for carcinogens from areca nut to act on the epithelium [6]. It could be assumed that the carcinogens accumulate over a long period of time either on or immediately below the epithelium allowing the carcinogens to act for a longer duration before it diffuses into submucosal tissues. Less vascularity may deny the quick absorption of carcinogens into the systemic circulation. Apart from this, the most controversial issue to deal is, how an epithelial malignancy develops in a predominantly connective tissue disorder? Recent evidence indicates that the cancer development and progression are facilitated by epithelial-mesenchymal interactions and transition. There are altered keratinocyte phenotypes, which predispose to development of malignancy in OSMF as a result of epithelial-mesenchymal interactions [7].

Tissue remodeling process is dependent on blood supply of the tissue. Angiogenesis is seen when there is increased requirement of nutrition [8]. Neoangiogenesis is a highly organized multistep process in which steps like vessel sprouting, endothelial cell migration, proliferation, tube formation and survival are involved [9]. Although angiogenesis cannot be measured directly, it can be inferred by quantification of vasculature, usually in the form of MVD [10], thus providing an index of angiogenesis [1].

The present systematic review was done to evaluate oral submucous fibrosis and its relation with stromal vascularity.

\section{MATERIAL AND METHODS}

\section{A. Search Strategy and inclusion criteria}

The study was approved by institutional review board of Riyadh Elm University with a registration no FRP/2019/183/38/40. The objective of this paper was to review the literature regarding oral submucous fibrosis and its relation with stromal vascularity. The search included published articles upto 2019 in English. The literature was searched using "PubMed" and electronic databases such as "Google Search". The following key words were used "oral submucous fibrosis," "vascularity", "epithelial atrophy". The search was restricted to English language literature. Titles and abstracts of appropriate studies were chosen. The first pool of primary studies and review articles were searched for references leading to additional papers missed 
in the computerized searches.

\section{B. Exclusion criteria}

Articles that were published in other languages other than English were excluded. Articles that had no data, insufficient data editorials, letters, case reports and historical reviews and in vitro studies were excluded.

\section{Results}

Using the established search strategy and inclusion criteria, 30 abstracts were identified (Fig. 1). Exclusion criteria included those studies with incomplete data (five), case reports (five) and animal studies (4). Based on the criteria a total 21 articles were included as shown in Table I [1]-[15], [19]-[21].

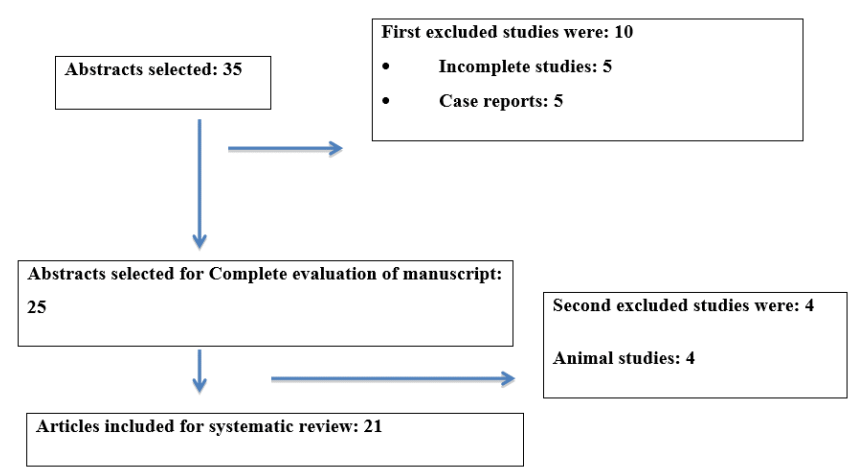

Fig. 1. Flow chart of article selection

TABLE I: STUDIES ON OSMF AND STROMAL VASCULARITY

Author

\section{Results}

Oral potentially malignant disorder represents altered cell populations characterized by neovascular response that is angiogenesis which is predictive of malignant potential

Tekade SA et al (2017) [20] MVD increased in stage I than in normal oral mucosa and reduced in stage II and III of OSMF. MVA and TVA reduced from normal mucosa to stage I, II and III of OSMF.

Murgod VV et al (2014) [19] MVD, MVA, MVLD increased from normal oral mucosa to OSMF to Oral squamouscellcarcinoma(OSCC). Vascularity increased in early stages and reduced in advanced stage of OSMF and increased in OSCC.

Singh et al (2010) [12] Koilocytes and collagen thickness increased with increasing stage of OSMF. Endothelial cell decreased with increasing stage of OSMF. MVA and MVLD increased in Stage II than Stage I and reduced in Stage III and Stage IV.

Desai SR et al (2010) [1] $\quad$ ET reduced from normal to Stage II, Stage IV to Stage III. MVD, MVLD, MVAP increased in Stage II and reduced in Stage III and Stage IV

Debnath et al (2013) ${ }^{13} \quad$ ET showed no statistical difference. MVD, MVA, MVLD decreased with increasing stage of OSMF. No correlation was found with trismus. The study concluded that dense fibrosis and decreased vascularity of the corium, in the presence of an altered cytokine activity, creates a

\begin{tabular}{ll}
\hline \hline & $\begin{array}{l}\text { unique environment for } \\
\text { carcinogens from both tobacco and } \\
\text { areca nut to act on the epithelium }\end{array}$ \\
\hline Rajendran R et al (2005) [14] & MVD was same in control and \\
& study group. MVAP and MVLD \\
increased with increasing stage of \\
OSMF. \\
\hline Fang CY et al (2000) [11] \\
MVD increased in early OSMF. \\
MVD, MVA and MVAP reduced \\
in middle and late stages of OSMF.
\end{tabular}

\section{Discussion}

OSMF is one of the collagen metabolic disorders. A number of possible mechanisms have been recommended in the pathogenesis of OSMF. It is believed to involve juxtaepithelial inflammatory response and fibrosis in the lamina propria, possibly due to increased cross-linking of collagen by upregulation of lysyl oxidase activity. This fibrosis, or the upsurge of collagen, results from the property of areca nut, which increases collagen assembly and also decreases collagen deprivation [11]. The most common early symptoms and signs are a burning sensation, blanching oral mucosa, ulceration and xerostomia. Later, the oral mucosa becomes stiff and opaque, by means of fibrous bands on the buccal mucosa, soft palate, lips, and tongue causing limit in mouth opening, intricacy in mastication, speech and swallowing [12]. The majority of the cases demonstrate epithelial atrophy with loss of rete ridges, epithelial atypia, and pigment incontinence. Lamina propria shows widespread fibrosis of collagen fibers with a chronic inflammatory cell infiltrate. In later stages, subepithelial hyalinization with atrophic changes in minor salivary glands and skeletal muscle can also be seen. OSMF does not revert spontaneously or on termination of areca nut chewing. Once the disease is evident, it either persists or becomes more severe with contribution of additional areas of the oral mucosa. OSMF is strongly connected with the risk of oral cancer. Studies suggest that dysplasia is seen in relation to $25 \%$ of biopsied OSMF cases [13].

\section{A. Studies Related to Oral Submucous Fibrosis and Vascularity}

Fang et al., Singh et al. and Debnath et al., morphometrically analyzed the mucosal vascularity in OSMF and concluded that microvessel hyperplasia occurs in early stages of OSMF and is markedly decreased in stage III and stage IV [14]- [16].

Desai et al., in their demonstration of increased vascularity by the presence of CD34 positive cells, hypothesized that they play an important role in tumor proliferation once the malignant transformation takes place [1].

Rajendran R et al., observed similar values of MVD in various stages of OSMF which were marginally low when compared to normal oral mucosa. They observed increase in Mean Vascular Area Percentage (MVAP) and Mean Vascular Luminal Diameter (MVLD) with progression of OSMF [17]. It is believed that stromal alteration is seen in OSMF and it progresses with the stage of OSMF [17].

The study put forward by Tilakaratne WM et al., that the dense fibrosis and decreased vascularity of the corium, in the presence of an altered cytokine activity, creates a unique 
environment for carcinogens from both tobacco and areca nut to act on the epithelium [18].

In previous studies by Murgod VV and Tekade SA et al on the mucosal vascularity in OSMF had found that the vascularity was increased in the early stages of OSMF and reduced in advanced stages, suggesting that inflammation plays a vital role in the early stages of the disease and progressive fibrosis predisposes to the atrophy of the epithelium and decrease vascularity, thereby causing subsequent malignant changes [19],[20].

Angiogenesis plays a vital role in wound healing process and as well as in growth and development. It is fundamental step in the transition of tumors from benign to malignant stage. Expression of angiogenesis property by pre neoplastic cell population predicts the malignant transformation of a premalignant condition [21].

\section{B. Limitations of the study}

Very fewer studies were found on relationship between OSMF and stromal vascularity. So, more detailed studies with larger sample size should be undertaken to clarify the relationship between the OSMF and stromal vascularity.

\section{CONCLUSION}

OSMF, a ubiquitous OPMD, is considered to be a collagen metabolic disorder. There are discernible alterations in the connective tissue secondary to chewing of areca nut, which further affects the surface epithelium. Studies show that vascularity was increased in early OSMF.

\section{REFERENCES}

[1] Desai SR, Mamatha GS, Khatri MJ, Shetty JS. Immunochemical expression of $\mathrm{CD} 34$ for characterization and quantification of mucosal vasculature and its probable role in malignant transformation of atrophic epithelium in oral submucous fibrosis. Oral Oncol. 2010; 46: $553-58$.

[2] Rajendran R. Oral submucous fibrosis: Etiology, pathogenesis, and future research. Bull World Health Organ. 1994;72: 985-96.

[3] Shieh TY, Yang JF. Collagenase activity in oral submucous fibrosis. Proc Natl Sci Counc Repub China B 1992; 16:106-10.

[4] Yang SF, Hsieh YS, Tsai CH, Chen YJ, Chang YC. Increased plasminogen activator inhibitor-1/tissue type plasminogen activator ratio in oral submucous fibrosis. Oral Dis 2007; 13:234-8.

[5] Rajendran R, Paul S, Mathews PP, Raghul J, Mohanty M. Characterization and quantification of mucosal vasculature in oral submucus fibrosis. Indian J Dent Res. 2005; 16:83-89.

[6] Tilakaratne WM, Klinikowski MF, Saku T, Peters TJ, Warnakuasuriya S. Oral submucous fibrosis: Review on etiology and pathogenesis. Oral Oncol 2006; 42:561-8.

[7] Lalli A, Tilakaratne WM, Ariyawardana A, Fitchett C, Leigh IM, Hagi-Pavli E, et al. An altered keratinocyte phenotype in oral submucous fibrosis: Correlation of keratin K17 expression with disease severity. J Oral Pathol Med 2008; 37:211-20.

[8] Takeda K, Hatamochi A, Ueki H. Increased number of mast cells accompany enhanced collagen synthesis in linear localized scleroderma. Arch Dermatol Res. 1989; 281:288-90.

[9] Sabarinath B, Sriram G, Saraswathi TR, Sivapathasundharam B. Immunohistochemical evaluation of mast cells and vascular endothelial proliferation in oral submucous fibrosis. Indian J Dent Res. 2011; 22:116-21.

[10] Hannen EJ, Riediger D. The quantification of angiogenesis in relation to metastasis in oral cancer: A review. Int J Oral Maxillofacial Surg. 2004; 33:02-07.

[11] Rajalalitha P, Vali S. Molecular pathogenesis of oral submucous fibrosis: A collagen metabolic disorder. J Oral Pathol Med 2005; 34:321-8.

[12] Auluck A, Rosin MP, Zhang L, Sumanth KN. Oral submucous fibrosis, a clinically benign but potentially malignant disease: Report of 3 cases and review of the literature. J Can Dent Assoc 2008; 74:735-40.

[13] Hazarey VK, Erlewad DM, Mundhe KA, Ughade SN. Oral submucous fibrosis: Study of 1000 cases from central India. J Oral Pathol Med 2007; 36:12-7.

[14] Fang CY, Han WN, Fong DY. A morphometric study on the microvessel in oral submucous fibrosis. Hunan Yi Ke Da Xue Xue Bao. 2000; 25:55-7.

[15] Singh M, Chaudhary AK, Pandya S, Debnath S, Singh M, Singh PA, et al. Morphometric analysis in potentially malignant head and neck lesions: Oral submucous fibrosis. Asian Pac J Cancer Prev. 2010; 11:257-60.

[16] Debnath S, Mitra B, Paul B, Saha TN, Maity A. Morphometric analysis of oral submucous fibrosis and its correlation with histological staging and clinical severity of trismus. Egypt J Ear Nose Throat Allied Sci. 2013; 14:85-90.

[17] Rajendran R, Paul S, Mathews PP, Raghul J, Mohanty M. Characterization and quantification of mucosal vasculature in oral submucus fibrosis. Indian J Dent Res. 2005; 16: 83-89.

[18] Tilakaratne WM, Klinikowski MF, Saku T, Peters TJ, Warnakulasuriya S. Oral submucous fibrosis: Review on aetiology and pathogenesis. Oral Oncol. 2006; 42: 561-8.

[19] Murgod VV, Kale AD, Angadi PV, Hallikerimath S. Morphometric analysis of the mucosal vasculature in oral submucous fibrosis and its comparison with oral squamous cell carcinoma. Journal of oral science. 2014; 56:173-78.

[20] Tekade SA, Chaudhary MS, Tekade SS, Sarode SC, Wanjari SP, Gadbail AR et al. Early stage oral submucous fibrosis is characterized by increased vascularity as opposed to advanced stages. Journal of clinical and diagnostic research. 2017; 11:92-96.

[21] Hande AH, Chaudhary MS, Gawande MN, Gadabali AR, Zade PR, Bajaj S, et al, Oral submucous fibrosis: An enigmatic morpho-insight. J Can Res Ther.2019;15: 463-9. 